\title{
The starfish story and lung transplantation for COVID-19
}

\author{
Dhruva Sharma $^{1}$ (D) Ganapathy Subramaniam ${ }^{2}$
}

Received: 14 October 2020 / Revised: 30 October 2020 / Accepted: 3 November 2020 / Published online: 24 November 2020

(C) Indian Association of Cardiovascular-Thoracic Surgeons 2020

To The Editor:

There is a famous parable:

"The story of the boy and the starfish"

One day a man walking along the beach notices a boy picking something carefully and throwing it gently back into the ocean. Approaching the boy, the man asks what he was doing. The boy replies: "throwing starfish back into the ocean". The surf is up and the tide is going out. If I do not throw them back, they'll die. The man said: "Son, don't you realize there are miles and miles of beach and hundreds of starfish? You can't make a difference!"

Till mid of October 2020, the coronavirus disease (COVID19) had killed more than 100,000 patients in India. The world estimates were approximately 10 times this number and the number of infected individuals was approximately $40-80$ times.

Acute respiratory distress syndrome (ARDS) coupled with severe acute respiratory syndrome coronavirus 2 (SARS-CoV2) infection has constituted a challenge in this worldwide pandemic of unrivalled consequence. Despite the majority of patients with COVID-19 presenting with mild or asymptomatic disease, $10 \%$ of cases necessitated intensive care unit (ICU) admission owing to the development of ARDS with a mortality rate of up to $60 \%$. Lung transplantation (LT) is considered a rescue therapy for patients with severe, treatment-refractory ARDS. Lung injury is usually present in around $30 \%$ of COVID-19 cases. Chest computed tomography (CT) scan dem-

Dhruva Sharma

drsharmadhruva1981@gmail.com

1 Department of Cardiothoracic and Vascular Surgery, SMS Medical College \& Attached Hospitals, J L N Marg,

Jaipur, Rajasthan 302001, India

2 Institute of Heart and Lung Transplant and Mechanical Circulatory Support, MGM Healthcare, No. 72, Nelson Manickam Road, Aminjikarai, Chennai 600029 Tamil-Nadu, India onstrated $97 \%$ and $75 \%$ sensitivity in diagnosing specimenpositive and specimen-negative patients, respectively, but with only $25 \%$ specificity. Of late, it is concluded that ground-glass opacity, with or without consolidation, was most commonly noted among COVID-19 patients. The COVID-19 Reporting and Data System (CO-RADS) grants a level of conjecture for pulmonary involvement of COVID-19, formed on the countenance seen at unenhanced chest $\mathrm{CT}[1,2]$.

In a case report by Lang et al. on LT for COVID-19linked ARDS [1], a 44-year-old woman tested positive for SARS-CoV-2 through nasopharyngeal swab real-time polymerase chain reaction (RT-PCR). Her respiratory condition worsened, requiring femoro-femoral veno-venous extracorporeal membrane oxygenation (ECMO). When not controlled medically, after 48 days, she was considered a possible candidate for LT. On pulmonary CT, there was evidence of complete consolidation of the lungs coupled with large necrotic areas and air inclusions, substantial infarctions of the peripheral parts of the lung parenchyma and thrombosis of small and medium-sized pulmonary arteries. Finally, a sequential bilateral LT was performed [1]. Surgeons from Chicago have also given a new set of lungs to a young woman with severe lung damage from COVID19 [3]. Another case was reported from Chennai, India, through media as a "triumphant completion of a critical bilateral lung transplant" on a 48-year-old COVID-19-positive patient. It was the first case of "successful completion of a critical bilateral lung transplant" on a COVID-19positive patient [4]. A case of double LT on a recovered COVID-19 patient was reported by media from Hyderabad, India [5].

A few intuitive indications and contraindications for LT can be derived from the limited experience worldwide. The patient should have had significant bilateral irreparable lung damage, and the patient should have single-organ involvement, preferably less than 65 years of age, with intact neurological status and sufficient muscle strength to participate in the extensive post-surgery rehabilitation process. LT should be performed by a team with sufficient experience in managing ECMO and high-risk LT [1]. 
Well to end the story, the boy picks up another starfish and throws it back into the surf. Thereafter, smiling at the man, the boy says: "I made a difference for that one". Considering the limited number of transplant patients worldwide, LT may not make a big difference in the overall COVID-19 outcomes. But, for the limited few, LT teams play the role of the boy, trying to gently throw the patient, who has been washed ashore during the pandemic, back into the ocean of life.

LT should therefore be added to the armamentarium of therapies for patients with COVID-19-related ARDS. However, patient selection criteria and timing of LT demand validation in further studies.

Funding This study was not funded.

\section{Compliance with ethical standards}

Conflict of interest The authors declare that they have no conflicts of interest.

Ethical approval Not applicable

Informed consent Not applicable

\section{References}

1. Lang C, Jaksch P, Hoda MA, et al. Lung transplantation for COVID19-associated acute respiratory distress syndrome in a PCR-positive patient. Lancet Respir Med. 2020. https://doi.org/10.1016/S22132600(20)30361-1.

2. Cypel M, Keshavjee S. When to consider lung transplantation for COVID-19. Lancet Respir Med. 2020;S2213-2600(20):30393-3. https://doi.org/10.1016/S2213-2600(20)30393-3.

3. Indian-origin doctor in U.S. performs double lung transplant for coronavirus survivor. UPDATED: JUNE 12, 2020Assessed on: September 18,2020, Available on site: [https://www.thehindu.com/ news/international/indian-origin-doctor-in-us-performs-doublelung-transplant-for-coronavirus-survivor/article31809789.ece].

4. Chennai doctors perform Asia's first lung transplant on Covid-19 patient. Updated: Aug 29, 2020, Assessed on: September 18,2020, Available on site : [https:/www.hindustantimes.com/india-news/ brain-dead-man-breathes-new-life-into-covid-19-patient-withsevere-lung-infection/story-UUVnm1 GL1Aqv4b1j9MILWN.html].

5. Doctors in Hyderabad perform India's first double lung transplant on recovered Covid patient. Updated 13 September, 2020, Assessed on September 18, 2020. Available from [https://theprint.in/health/ doctors-in-hyderabad-perform-indias-first-double-lung-transplanton-recovered-covid-patient/501590/].

Publisher's note Springer Nature remains neutral with regard to jurisdictional claims in published maps and institutional affiliations. 